



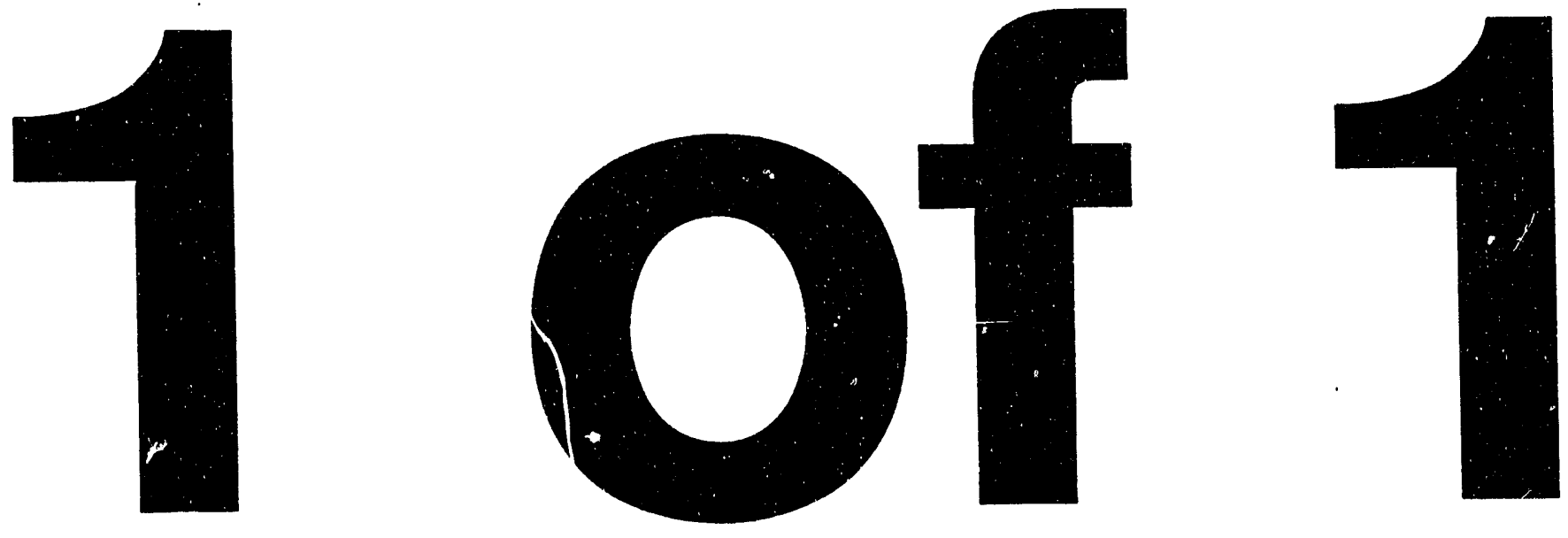


\section{Performance of the SLC Polarized Electron Source with High Polarization*}

J. E. Clendenin, R. K. Alley, H. Aoyagi, † J. C. Frisch, C. L. Garden, E. W. Hoyt, R. E. Kirby, L. A. Klaisner, A.V. Kulikov, C.Y. Prescott, P. J. Sácz, D. C. Schult, H. Tang, J. L. Tumer, M. Woods, A. D. Yeremian, and M. S. Zolotorev

Stanford Linear Accelerator Center, Stanford University, Stanford, CA 94309

\section{Abstract}

For the 1992 operating cycle of the SLAC Lincar Collider (SLC), the polarized electron source (PES) during its maiden run successfully met the pulse intensity and overall efficiency requirements of the SLC. However, the polarization of the bulk GaAs cathode was low $(-27 \%)$ and the pulse-to-pulse stability was marginal. We have shown that adequate charge for the SLC can be extracted from a strained layer cathode having $\mathrm{P}_{\mathrm{e}} \sim 80 \%$ even though the quantum efficiency (QE) is $<1 \%$. The recent addition of a separate chamber to the PES - which allows cathodes to be loaded into the gun after the vacuum bake and after high voltage ( $\mathrm{HV}$ ) processing without breaking vacuum-increases the reliability for achieving an adequate photoclectron yield. A new SLAC-built pulsed Ti:sapphire laser permits operation of the PES at the required wavelength with sufficient power to fully saturate the yield, and thus improve the $\mathrm{e}^{-}$beam stability. The performance of the PES during the 1993 SLC operating cycle with these and other improvements is discussed.

\section{INTRODUCTION}

In 1992, during the first SLC run with a polarized bcam, the SLC performance was an impressive improvement over previous years. The luminosity of the accelerator and the efficiency of the detector were improved sufficiently for the SLAC Large Detector (SLD) to $\log$ over $10,000 \mathrm{Z}^{\circ} \mathrm{S}$ with an average electron polarization at the Interaction Point (IP) of $23 \%$ during a run of approximately four months. The average (best) luminosity achieved by the end of the run was 18 (25) $\mathrm{Z}^{\circ}$ /hour. The goals for 1993 were to achieve at least 50,000 $\mathrm{Z}$ s on tape with an electron polarization $>35 \%$ at the IP. To meet these goals, it was estimated that the average (peak) luminosity should be about 23 (30) $\mathrm{Z}^{\circ}$ /hour. As shown in Table 1, the SLC/SLD are well on the way to meeting these goals. As will be discussed in more detail below, the polariza-

\begin{tabular}{|c|c|c|c|c|c|}
\hline \multicolumn{6}{|c|}{ Table 1. SLC/SLD Performance/Goals } \\
\hline & \multicolumn{3}{|c|}{ Performance } & \multicolumn{2}{|c|}{1993} \\
\hline & 1991 & \multicolumn{2}{|c|}{1992} & Goals & Performance \\
\hline $\mathrm{Z}^{\circ} \mathrm{h}$ typical & & & 18 & 23 & 35 \\
\hline $\mathrm{Z}^{\circ} / \mathrm{h}$ peak & & & 25 & 30 & 45 \\
\hline $\mathrm{Z}^{\circ} /$ day peak & & & 400 & 550 & 600 \\
\hline$Z^{\circ}$ total & 370 & $1 \mathrm{~K}$ & $11 \mathrm{~K}$ & $>50 \mathrm{~K}$ & \\
\hline Pe@IP & 0 & 0 & $23 \%$ & $>35 \%$ & $-60 \%$ \\
\hline Pe@Source & 0 & 0 & $27 \%$ & & $\sim 80 \%$ \\
\hline
\end{tabular}

Fermanent address: Faculty of Science, Nagoya University, Nagoya 464-01. Japan.

*Work supported by Department of Energy contract DE-AC03. 76 SF00515. tion has been increased to $>60 \%$ at the IP, and the average (peak) luminosity is now about $35(45) \mathrm{Z}^{\circ} \mathrm{h}$, or in excess of $500 \mathrm{Z} /$ day.

For 1993, the SLC peak luminosity has been improved primarily by reducing the betatron coupling in each of the Damping Rings (DR) to produce flat beams. In addition, the average luminosity has been given an added boost by careful attention to reliability details, leading to a significant increase in efficiency. To improve the polarization, the energy of the $\mathrm{e}^{-}$DR was increased to produce the correct spin precession to the linac-to-ring (LTR) spin rotator, thus reducing a $5 \%$ (relative) depolarization effect to $\sim 2 \%$. However, the primary reason for the increased polarization of the electron beam is the inaugu-ration in the PES of specially designed cathodes that remove the degeneracy at the $\Gamma$ point between the heavyhole and light-hole valence bands. Photoexcitation of such cathodes, using circularly polarized light of the proper wavelength, produces highly polarized electrons.

\section{CATHODES FOR HIGH POLARIZATION}

The theoretical upper limit for the polarization of photoelectrons from bulk-grown NEA GaAs is $50 \%$. Typically, the actual polarization is much lower unless very thin active layers or cryogenic temperatures are used. For 1992, the SLC PES operated with a bulk GaAs cathode at room temperature, resulting in a polarization at the source of only $27 \%$ [1].

Highly polarized electrons from photocathodes have been the focus of efforts at SLAC and other laboratories for many years. The first demonstration of success was at SLAC [2] in 1991 , followed soon thereafter by similar results at Nagoya [3]. The technique employed is to introduce a uniaxial tensile strain in the growth direction by growing the active layer on a substrate with an appropriately smaller lattice constant. Above a certain critical thickness, the strain thus induced will decrease, so the active layer must be kept no thicker than a few hundred nanometers.

A crystal cut from an MOCVD-grown [4] 2-inch diameter wafer having a $300-\mathrm{nm}$ active (strained) layer of $\mathrm{GaAs}(100)$, $\mathrm{Zn}$-doped to $5 \times 10^{18} \mathrm{~cm}^{-3}$ and grown on a $2.5 \mu \mathrm{m}$ p-GaAs 0.76 $\mathrm{P}_{0.24}$ sublayer, was measured at SLAC to have a peak polarization of $\sim 80 \%$ at $\sim 860 \mathrm{~nm}$ (room temperature) [5,6]. However, the low intensity QE measured at low voltage was only $-0.1 \%$, which gave rise to concern that it might be difficult to extract the high peak charge needed for the SLC ( $10 \mathrm{nC}$ per microbunch in the present mode of operation).

A second crystal cut from the same wafer was tested at HV in the SLC gun [7] in a laboratory system having a high power laser tuned to $850 \mathrm{~nm}$, but at present having no polarimeter. The saturated charge (i.e., the maximum charge for high laser power) was found to scale linearly with

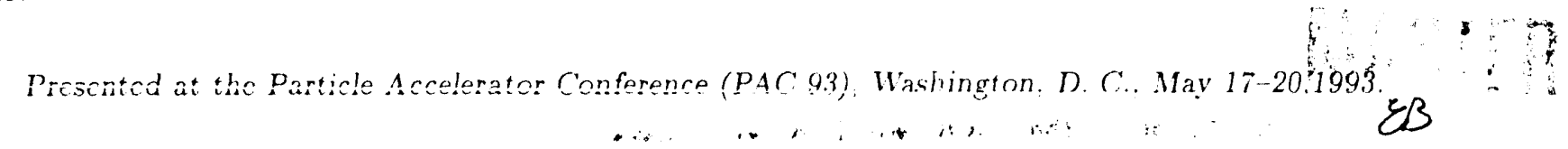




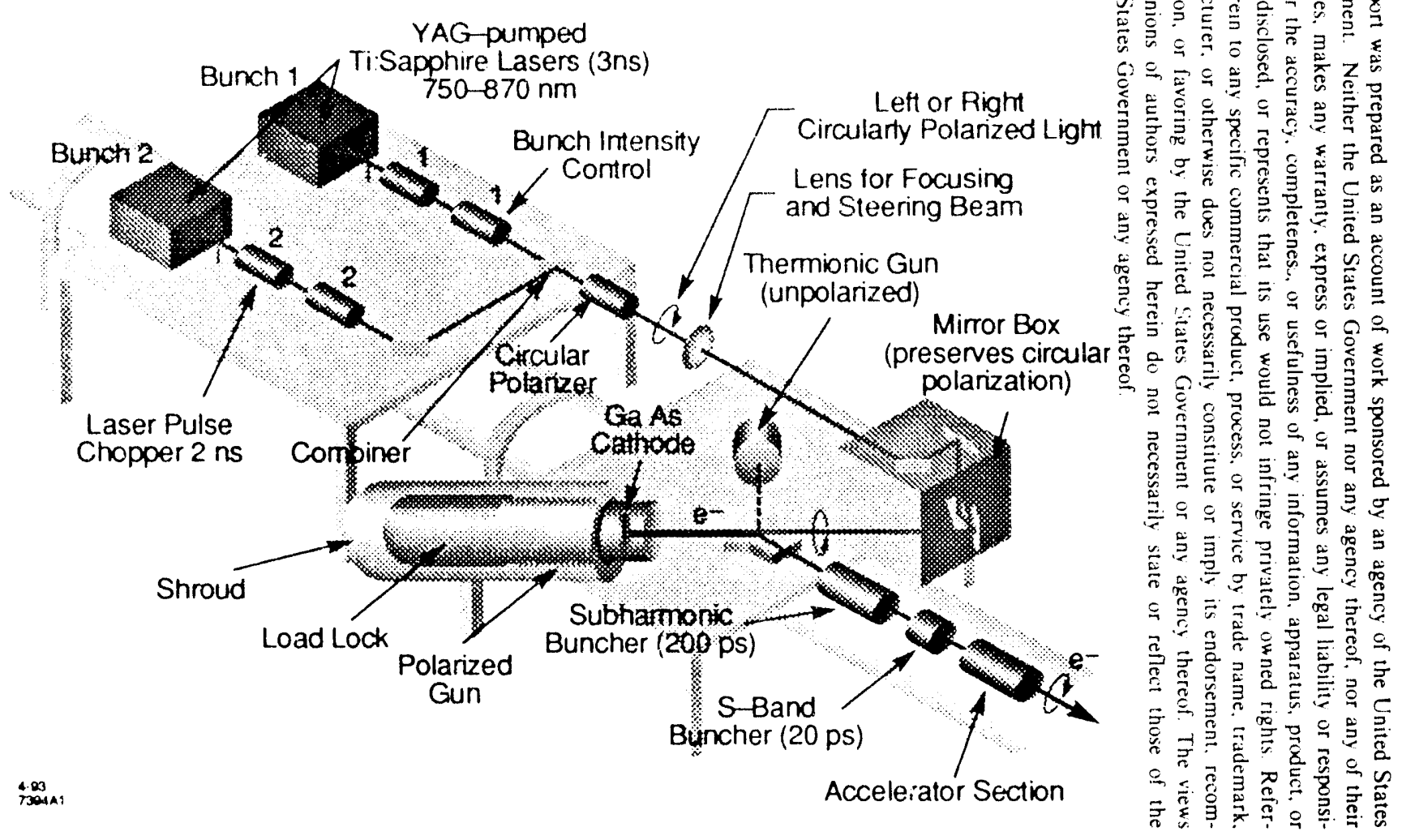

Figure 1. Schematic of the SLC PES for the 1993 SLC/SLD run.

cathoxic hias, whereas for a low intensity laser, the log of the photocurrent scaled as the square root of the voltage (the expected Schottky effect) [8]. A saturated charge of $19 \mathrm{nC}$, very close $w$ the space charge limit of $\sim 21 \mathrm{nC}$, was achieved at $120 \mathrm{kV}$ when the low intensity $\mathrm{QE}$ was $1 \%$ measured at 830 $\mathrm{nm}$ and $120 \mathrm{kV}$. The improvement in QE was probably due to the use of a load-lock system, as described later. This crystal, together with a third from the same wafer was in the cathode tray of the load lock when the polarized source was installed at the SLC for the 1993 run.

\section{RECENT POLARIZED ELECTRON SOURCE IMPROVEMENTS}

The pulsed dye laser used in the 1992 run was limited by power and lifetime requirements to $715 \mathrm{~nm}$, a wavelength too low to permit any polarization gain with the new cathodes. A new Nd:YAG pumped Ti:sapphire laser, Q-switched and cavity-dumped, designed and built at SLAC [9], was installed for this run. This laser, together with associated beam transport optics, provides up to $1(00 \mu \mathrm{J}$ energy at the cathode $\mathrm{t}$. each $2.0 \mathrm{~ns}$ FWHM micropulse at wavelengths up to $870 \mathrm{~nm}$.

The addition of a load lock that allows a photocathode to be introduced into the gun chamber after the chamber is baked and HV processed has proven to be a major step in improving the PES performance and reliability [10]. Up to four cathodes can be stored at one time in the load-lock system.

The cathodes are activated in the load-lock system, in pan to avoid $\mathrm{Cs}$ contamination in the gun itself. Cathode activation and/or switching of cathodes takes about 8 hours, followed by a period of 1(2) 20 hours in which the gun shroud is purged to remove water vapor before operating at HV. For simple addition of small amounts of $\mathrm{Cs}$ to the operating cathode, $\mathrm{Cs}$ channels [11] that encircle the $\mathrm{e}^{-}$beam are located in the gun chamber just downstream of the anode. The $\mathrm{Cs}$ channels are operated remotely through the SLC Control Program (SCP). A schematic of the present PES is shown in Fig. 1. A cross section of the gun is shown in Ref. [12].

\section{1993 POLARIZED ELECTRON SOURCE PERFORMANCE}

The polarization of the $\mathrm{e}^{-}$beam could be measured at $50 \mathrm{GeV}$ by a MOller polarimeter at the end of the linac and by a Compton polarimeter at the IP. The latuer was sensitive to the tuning of the Ares. The wavelength of the PES laser and the Compton polarization were monitored continuously. A sample is shown in Fig. 2. Combined with a MØller measure ment made early in the run with the $e^{-}$DR bypassed, the source polarization was found to peak at $\sim 865 \mathrm{~nm}$ (cathode at

7) with $\mathrm{Pe}-80 \%$

In its first 10 weeks of operation with a strained layer cathode, the PES was able to provide a highly polarized $\mathrm{e}^{-}$ beam for the SLC $>95 \%$ of the time. During the run, the charge limit effect [13] was not a serious limitation [14], although the low-tensity QE following a cesiation touchup was only $-(0.5 \%$ measured at $830 \mathrm{~nm}$ and $120 \mathrm{kV}$.

The QE was monitored by detecting (using a nanoam-meter attached to the HV electrode) the photocurrent drawn 
from the cathode by a modulated $830 \mathrm{~nm}$ diode laser (gated off during the beam pulse) attached to a side window of the gun. The nanoammeter was powered and also connected to the SCP through an optical link.

The $\mathrm{e}^{-}$intensity was controlled through a feedback system that varied " " size of the Ti:sapphire laser spot on the cathode. The laser itself was operated at very high energy so as to keep the cathode always completely saturated. In this manner the $\mathrm{e}^{-}$pulse-to-pulse intensity jitter could be kept $-1 \%$, although the laser energy jitter was often $>3 \%$. The charge limit did reduce the maximum charge available as a function of cathode surface conditions.

The overall pressure in the gun was $\sim 10^{-11} \mathrm{Torr}$, dominated by $\mathrm{H}_{2}$. As shown in Fig. 3, the rate of decrease of the low intensity $\mathrm{QE}$ was not affected the charge extracted frorn the gun. The $\mathrm{QE}$ was found to decrease by $<0.05 \% /$ day. The cathode lifetime was probably extended in this vacuum system by operating the cathode at $-6^{\circ} \mathrm{C}$ and by the success of the HV processing that reduced the dark current (cathode current at
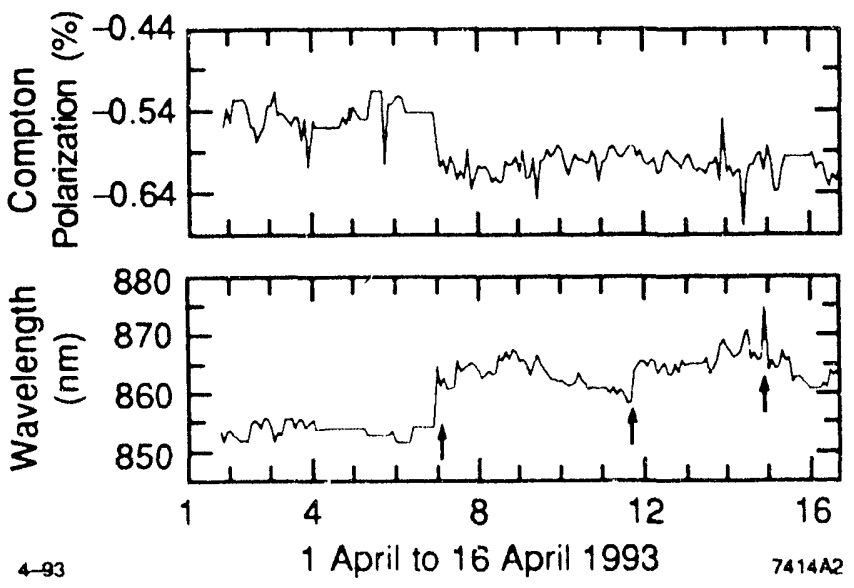

Figure 2. Comparison of PES laser wavelength and Compton polarization over a period of 16 days. The PES wavelength was deliberately changed on April 6, 11, and 14: $853 \rightarrow 865 \mathrm{~nm}, 859 \rightarrow 864 \mathrm{~nm}$, and $870 \rightarrow 864 \mathrm{~nm}$, respectively.
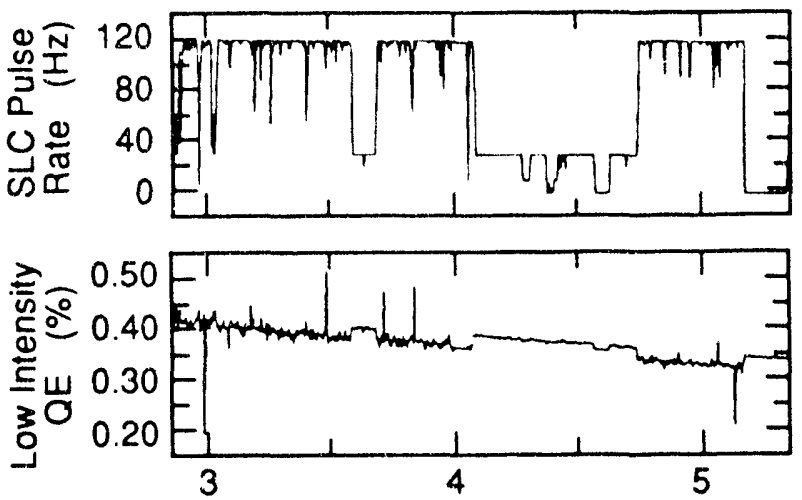

2 April to 5 April 1993

Figure 3. Comparison of the low-intensity QE monitored through a period of $\sim 15$ hours when the SLC macropulse rate was dropped from the usual $120 \mathrm{~Hz}$ to $30 \mathrm{~Hz}$. The offset in the QE is probably an artifact of the instrumentation.
$120 \mathrm{kV}$ with all lasers blocked) to $<50 \mathrm{nA}$ [15]. To maintain the required charge, cesium was added in intervals of -3 days.

It was a simple matter to cesiate from the SLC Control Center using the SCP to lower the HV to $1 \mathrm{kV}$, then to operate the channel cesiator at a fixed $4 \mathrm{~A}$ for -4 minutes. To extend the cesiation intervals, the cathode was usually cesiated until the photocurrent dropped $-20 \%$ lower than its peak value. The whole operation took $\sim 25$ minutes.

\section{NEXT DEVELOPMENTS}

Three new developments may improve the source performance even further in the near future. One of the four SLC polarized guns has been modified to double the area of the semiconductor cathode in order to increase the charge limited current. This will be tested soon in the laboratory. Operating the gun at $150 \mathrm{kV}$ instead of $120 \mathrm{kV}$ should also increase the photoelectron emission $25 \%$ when charge limited.

Source polarizations $>90 \%$ can be achieved with even thinner strained-layer cathodes [6]. The charge limit for such cathodes is under investigation.

Within the next few months, a Mott polarimeter will be added to the SLC high beam intensity testing system allowing a crystal to be fully qualified for $P_{e}, Q E$, charge limit, and lifetime before this same crystal is installed in the operating SLC gun.

\section{REFERENCES}

[1] D. Schultz et al., "Polarized Source Performance in 1992 for SLC-SLD," presented at the 10th Int. Sym. on H.E. Spin Physics, Nagoya, Nov. 9-14, 1992.

[2] T. Maruyama et al., Phys. Rev. Lett. 66 (1991) 2376.

[3] T. Nakanishi et al., Phys. Lett. A 158 (1991) 345.

[4] SPIRE Corp., 1 Patriots Park, Bedford, MA 01730.

15] T. Maruyama et al., "Data for Spire $0.3 \mathrm{~mm}$ strained GaAs (MO5-1684)," SLAC internal report (1993), unpublished.

[6] T. Maruyama et al., Phys. Rev. 846 (1992) 4261.

[7] Active area of SLC cathode has a $14 \mathrm{~mm}$ diameter.

[8] H. Tang et al., "Observation of Non-Linear Photoemission Effects from III-IV Semiconductors," these proceedings.

[9] J. Frisch et al., "Operation of the New SLC Polarized Electron Source Laser," these proceedings.

[10] R.E. Kirby et al., "An In-Situ Photocathode Loading System for the SLC Polarized Electron Gun," these proceedings.

[11] Two SAES C's channels each with $17 \mathrm{~mm}$ active length connected in series.

[12] D.C. Schulu et al., Proc. of the Third European Part. Acc. Conf., Berlin (1992) 1029.

[13] M. Woods et al., SLAC-PUB-5894 (1992), to be published in J. Appl. Phys.

[14] The two microbunches required for the SLC were actually produced by separate Ti:sapphire cavities. To minimize charge limiting effects for the second unpolarized microbunch, its laser was tuned to $770 \mathrm{~nm}$

[15] P. Sácz et al., "High Voltage Processing of the SLC Polarized Electron Gun," these proceedings. 

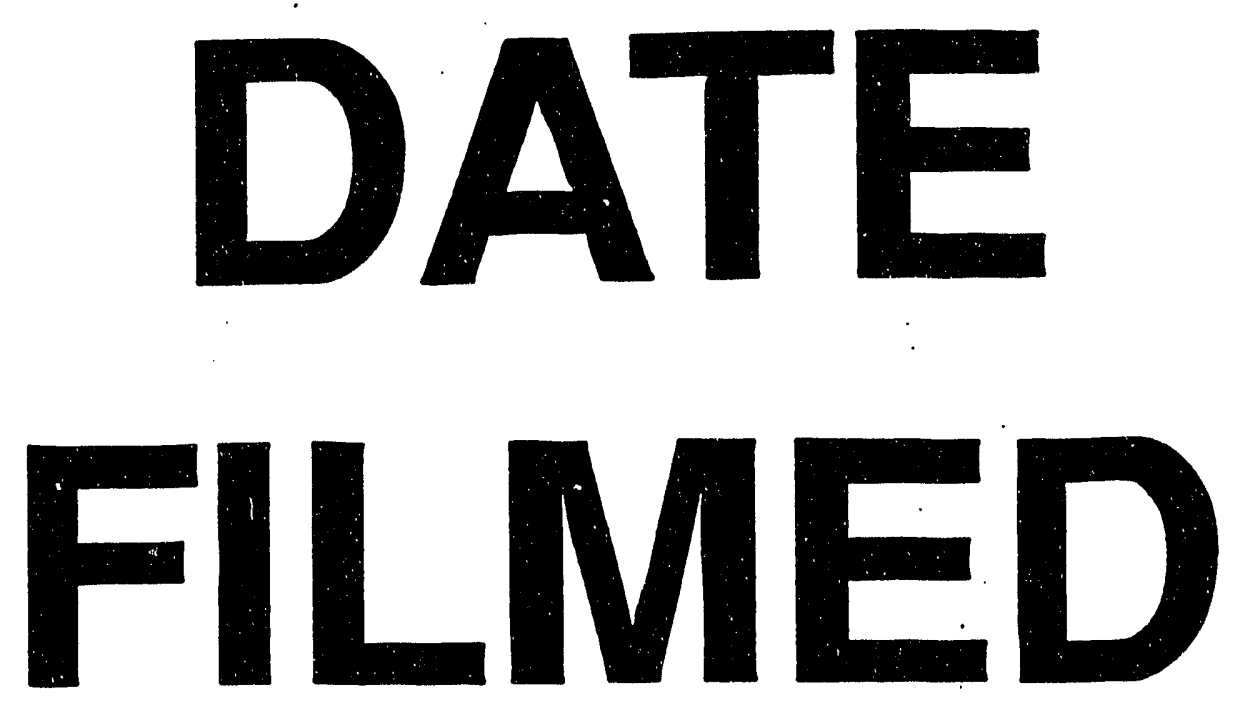

$11 / 19 / 93$
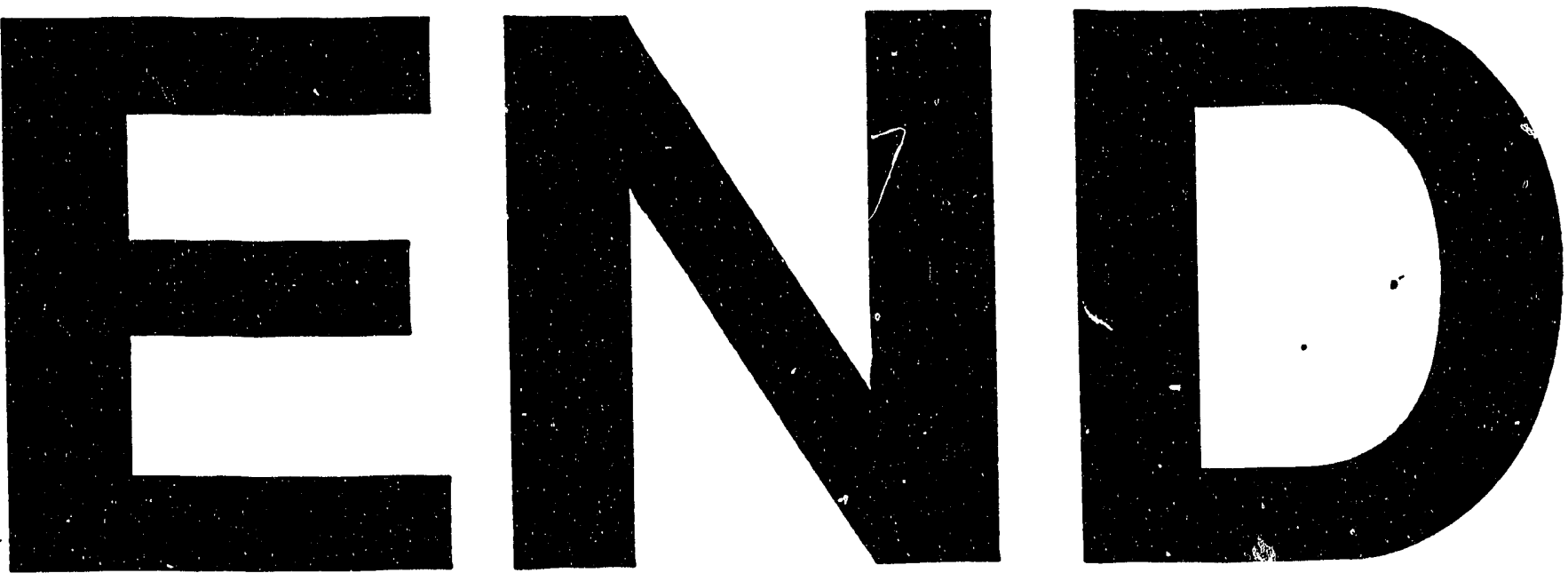
